\title{
SOME INVARIANTS OF CERTAIN PAIRS OF HYPERSURFACES
}

\section{CHUAN-CHIH HSIUNG}

Introduction. It is known $[8,9]^{1}$ that if two surfaces in ordinary space have a common tangent plane at an ordinary point, then the ratio of their total curvatures at this point is a projective invariant, and the theorem holds true similarly for hyperspaces. ${ }^{2}$ In connection with this theorem and the investigation of Bouton [2], Buzano [3] and Bompiani [1] have shown the existence of a projective invariant, together with metric and projective characterizations, determined by the neighborhood of the second order of two surfaces $S, S^{*}$ at two ordinary points $O, O^{*}$ in ordinary space under the conditions that the tangent planes of the surfaces $S, S^{*}$ at the points $O, O^{*}$ be distinct and have $O O^{*}$ for the common line. Furthermore, the other case in which the tangent planes of the surfaces $S, S^{*}$ at the points $O, O^{*}$ are coincident ${ }^{3}$ has been considered in recent papers of the author [6, 7].

It is the purpose of the present paper to generalize the results of the two cases mentioned above.

Let $V_{n-1}, V_{n-1}^{*}$ be two hypersurfaces in a space $S_{n}$ of $n$ dimensions, and $t_{n-1}, t_{n-1}^{*}$ the tangent hyperplanes of the hypersurfaces $V_{n-1}, V_{n-1}^{*}$ at two ordinary points $O, O^{*}$. For the subsequent discussion it is convenient to assume in Chapter $\mathrm{I}$ that the tangent hyperplanes $t_{n-1}, t_{n-1}^{*}$ are coincident. We can $(\$ 1)$, as in ordinary space, determine a projective invariant by the neighborhood of the second order of the hypersurfaces $V_{n-1}, V_{n-1}^{*}$ at the points $O, O^{*}$; and the projective and metric characterizations of this invariant are given in the next two sections.

Chapter II treats of the case in which the tangent hyperplanes $t_{n-1}, t_{n-1}^{*}$ are distinct, and the common tangent flat space $t_{n \rightarrow 2}$ of $t_{n-1}, t_{n-1}^{*}$ contains the line $O O^{*}$. We first $(\$ 4)$ show by analysis the existence of two projective invariants determined by the neighbor-

Presented to the Society, February 26, 1945; received by the editors October 3, 1944, and, in revised form, March 19, 1945.

${ }^{1}$ Numbers in brackets refer to the bibliography at the end of the paper.

2 The simple projective characterizations of this invariant were given by C. Segre [10] for two plane curves and by P. Buzano [4] for two surfaces in space $S_{n}(n>2)$. On the other hand, A. Terracini [11] also interpreted projectively this invariant by virtue of the conception of density of dualistic correspondences.

${ }^{3}$ It should be noted that for two plane curves having a common tangent at two ordinary points no projective invariant can be determined by the neighborhood of the second order of the two curves at these points. See my paper [5]. 
hood of the second order of the hypersurfaces $V_{n-1}, V_{n-1}^{*}$ at the points $O, O^{*}$; and then $(\$ \$ 5,6)$ give them simple projective and metric characterizations. From the fact that one of the two invariants is reduced to 1 when the immersed space $S_{n}$ is of three dimensions, it follows that our result in this chapter stands actually for a generalization of that of Buzano and Bompiani.

\section{Chapter I. Two hypersurfaces With COMMON TANGent HYPERPLANE AT TWO ORDINARY POINTS}

1. Derivation of an invariant. Let $V_{n-1}, V_{n-1}^{*}$ be two hypersurfaces in a space $S_{n}$ of $n$ dimensions with common tangent hyperplane $t_{n-1}$ at two ordinary points $O, O^{*}$. Let $x_{1}, \cdots, x_{n+1}$ denote the homogeneous projective coordinates of a point in the space $S_{n}$. If we choose the points $O, O^{*}$ to be the vertices $(1,0, \cdots, 0),(0, \cdots, 0,1,0)$ of the system of reference, and the common tangent hyperplane $t_{n-1}$ to be the coordinate hyperplane $x_{n+1}=0$ of the system, then the power series expansions of the hypersurfaces $V_{n-1}, V_{n-1}^{*}$ in the neighborhood of the points $O, O^{*}$ may be written in the form

$$
\begin{aligned}
& V_{n-1}: \frac{x_{n+1}}{x_{1}}=\sum_{i, k=2}^{n} l_{i k} \frac{x_{i}}{x_{1}} \frac{x_{k}}{x_{1}}+\cdots, \\
& V_{n-1}^{*}: \frac{x_{n+1}}{x_{n}}=\sum_{i, k=1}^{n-1} m_{i k} \frac{x_{i}}{x_{n}} \frac{x_{k}}{x_{n}}+\cdots .
\end{aligned}
$$

In order to find a projective invariant of the hypersurfaces $V_{n-1}$, $V_{n-1}^{*}$ at the points $O, O^{*}$, we have to consider the most general projective transformation of coordinates which shall leave the points $O, O^{*}$ and the hyperplane $t_{n-1}$ unchanged:

$$
\begin{aligned}
x_{i} & =\sum_{r=1}^{n+1} a_{i r} x_{r}^{\prime} \quad(i=1, \cdots, n), \\
x_{n+1} & =a_{n+1, n+1} x_{n+1}^{\prime},
\end{aligned}
$$

where

$$
\begin{gathered}
a_{21}=\cdots=a_{n 1}=0, \quad a_{1 n}=\cdots=a_{n-1, n}=0, \\
D=\left|\begin{array}{cccc}
a_{22} & a_{23} & \cdots & a_{2, n-1} \\
a_{32} & a_{33} & \cdots & a_{3, n-1} \\
\cdot & \cdot & \cdot & \cdot \\
a_{n-1,2} & a_{n-1,3} & \cdots & a_{n-1, n-1}
\end{array}\right| \neq 0 .
\end{gathered}
$$

The effect of this transformation on equations (1), (2) is to produce 
two other equations of the same form whose coefficients, indicated by accents, are given by the formulas

$$
\begin{array}{rlrl}
a_{11} a_{n+1, n+1} l_{i k}^{\prime} & =\sum_{r, a-2}^{n} a_{r i} a_{s k} l_{r s} & (i, k=2, \cdots, n), \\
a_{n n} a_{n+1, n+1} m_{i k}^{\prime}=\sum_{r, s=1}^{n-1} a_{r i} a_{s k} m_{r e} & (i, k=1, \cdots, n-1) .
\end{array}
$$

From equations (4), (5), (6) it is easily seen that the determinants

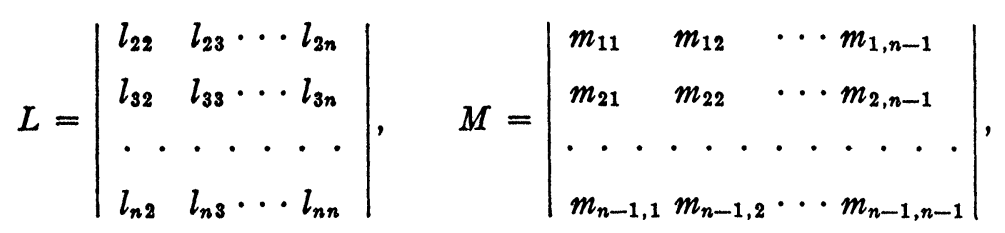

and their transformed ones $L^{\prime}, M^{\prime}$ are connected by the relations

$$
\begin{aligned}
a_{11}^{n-1} a_{n+1, n+1}^{n-1} L^{\prime} & =a_{n n}^{2} D^{2} L, \\
a_{n n}^{n-1} a_{n+1, n+1}^{n-1} M^{\prime} & =a_{11}^{2} D^{2} M .
\end{aligned}
$$

Further elimination of $a_{i k}$ from equations (6), (7) shows immediately that the quantity

$$
I=\frac{L}{M}\left(\frac{m_{11}}{l_{n n}}\right)^{(n+1) / 3}
$$

is a projective invariant determined by the neighborhood of the second order of the hypersurfaces $V_{n-1}, V_{n-1}^{*}$ at the points $O, O^{*}$.

2. A projective characterization of the invariant $I$. Let the polar spaces of the line $O O^{*}$ with respect to the asymptotic hypercones of the hypersurfaces $V_{n-1}, V_{n-1}^{*}$ at the points $O, O^{*}$ be respectively denoted by $t_{n-2}, t_{n-2}^{*}$, which determine a space $t_{n-3}$ of $n-3$ dimensions in the common tangent hyperplane $x_{n+1}=0$. If the $n-2$ vertices, other than $O$ and $O^{*}$, of the system of reference in the hyperplane $x_{n+1}=0$ be chosen in the space $t_{n-8}$, then the invariant $I$ may be reduced to

$$
I=\frac{L_{n n}}{M_{11}}\left(\frac{m_{11}}{l_{n n}}\right)^{(n-2) / 3},
$$

where $L_{n n}, M_{11}$ are the minors of $l_{n n}, m_{11}$ in the determinants $L, M$ respectively.

For the purpose of finding a projective characterization of the in- 
variant $I$ we first observe the space $S_{\mathbf{z}}$ determined by the vertices $(1,0, \cdots, 0),(0, \cdots, 0,1,0),(0, \cdots, 0,1)$ and any one, say for instance $O_{2}(0,1,0, \cdots, 0)$, of the system of reference in the space $t_{n-8}$. The space $S_{3}$ intersects the hypersurfaces $V_{n-1}, V_{n-1}^{*}$ in two surfaces $S, S^{*}$. Since the tangent planes of the surfaces $S, S^{*}$ at the points $O, O^{*}$ are coincident we have a projective invariant, denoted by $J$,

$$
J=\frac{l_{22}}{m_{22}}\left(\frac{m_{11}}{l_{n n}}\right)^{1 / 8},
$$

whose projective characterization has been obtained [6].

Let $Q\left(Q^{*}\right)$ be any quadric in the space $S_{3}$ which has $\mathrm{OO}_{2}\left(\mathrm{O}^{*} \mathrm{O}_{2}\right)$, $0 O^{*}\left(O O^{*}\right)$ for generators and whose curve of intersection with the element of the second order of the surface $S\left(S^{*}\right)$ at the point $O\left(O^{*}\right)$ has a cusp at $O\left(O^{*}\right)$. If the cone projecting from the point $O_{2}$ the curve of intersection of the two quadrics $Q, Q^{*}$ be tangent to the common tangent plane $O \mathrm{O}^{*} \mathrm{O}_{2}$ along a line through the point $\mathrm{O}_{2}$, then this line must be one of the lines (cf. [6])

$$
\begin{aligned}
& x_{n} \pm( \pm 1)^{1 / 2}\left(\frac{m_{11} m_{22}}{l_{22} l_{n n}}\right)^{1 / 4} x_{1}=0, \\
& x_{3}=\cdots=x_{n-1}=x_{n+1}=0 .
\end{aligned}
$$

We may now uniquely determine a point $P$ on the line $O O^{*}$ such that the cross ratio of the three points $O, O^{*}, P$, and the intersection of the line (11) with $O O^{*}$ is equal to $J^{1 / 4}$. On the other hand, the asymptotic hypercones of the hypersurfaces $V_{n-1}, V_{n-1}^{*}$ at the points $O, O^{*}$ determine a pencil of hyperquadrics in the hyperplane $x_{n+1}=0$, among which there exist $n$ hypercones, two of them being the asymptotic hypercones. The line $O O^{*}$ intersects each of the other $n-2$ hypercones in a pair of points. Let $Q_{i}(i=1, \cdots, n-2)$ be any one of each pair of these points and $D_{i}$ the cross ratio of the four points $O, O^{*}, Q_{i}, P$ on the line $0 O^{*}$, then we may easily show that the invariant I can be expressed in terms of the $n-2$ cross ratios $D_{1}, D_{2}, \cdots, D_{n-2}$ as follows:

$$
I=( \pm 1)^{n-2}\left(D_{1} D_{2} \cdots D_{n-2}\right)^{2} .
$$

3. A metric characterization of the invariant $I$. It is deemed worth while to give in this section a simple metric characterization of the invariant $I$. For this purpose we choose an orthogonal Cartesian coordinate system in such a way that the point $O$ be the origin, the line $O O^{*}$ be the $X_{n-1}$-axis, and the common tangent hyperplane $t_{n-1}$ be the coordinate hyperplane $X_{n}=0$. Then the power series expan- 
sions of the hypersurfaces $V_{n-1}, V_{n-1}^{*}$ in the neighborhood of the points $O, O^{*}$ may be put into the form

$$
\begin{aligned}
V_{n-1}: X_{n}= & \sum_{i, k=1}^{n-1} \lambda_{i k} X_{i} X_{k}+\cdots \\
V_{n-1}^{*}: X_{n}= & \sum_{i, k=1}^{n-2} \mu_{i k} X_{i} X_{k}+2 \sum_{i=1}^{n-2} \mu_{i, n-1} X_{i}\left(X_{n-1}-h\right) \\
& +\mu_{n-1, n-1}\left(X_{n-1}-h\right)^{2}+\cdots,
\end{aligned}
$$

where $h$ is the distance between the points $O, O^{*}$.

Let $y_{0}, y_{1}, \cdots, y_{n}$ be the homogeneous coordinates of a point defined by the formulas

$$
X_{i}=y_{i} / y_{0} \quad(i=1, \cdots, n),
$$

and let us consider the most general projective transformation of coordinates which shall leave the point $O$ and the common tangent hyperplane $t_{n-1}$ invariant, and change the point $O^{*}$ into the vertex $(0, \cdots, 0,1,0)$ of the new coordinate system:

where

$$
\begin{aligned}
y_{0} & =\sum_{i=0}^{n} a_{0 i} y_{i}^{\prime}, \\
y_{i} & =\sum_{r=1}^{n} a_{i r} y_{r}^{\prime} \quad(i=1, \cdots, n-1), \\
y_{n} & =a_{n n} y_{n}^{\prime},
\end{aligned}
$$

$$
\begin{aligned}
& a_{1, n-1}=\cdots=a_{n-2, n-1}=0, \quad a_{n-1, n-1}=h a_{0, n-1}, \\
& \Delta=\left|\begin{array}{cccc}
a_{11} & a_{12} & \cdots & a_{1, n-2} \\
a_{21} & a_{22} & \cdots & a_{2, n-2} \\
\cdots & \cdot & \cdots & \cdot \\
a_{n-2,1} & a_{n-2,2} & \cdots & a_{n-2, n-2}
\end{array}\right| \neq 0 .
\end{aligned}
$$

By transformations (15) and (16), equations (13), (14) shall be carried into two others of the form

$$
\begin{aligned}
& V_{n-1}: \frac{y_{n}^{\prime}}{y_{0}^{\prime}}=\sum_{i, k=1}^{n-1} p_{i k} \frac{y_{i}^{\prime}}{y_{0}^{\prime}} \frac{y_{k}^{\prime}}{y_{0}^{\prime}}+\cdots, \\
& V_{n-1}^{*}: \frac{y_{n}^{\prime}}{y_{n-1}^{\prime}}=\sum_{i, k=0}^{n-2} q_{i k} \frac{y_{i}^{\prime}}{y_{n-1}^{\prime}} \frac{y_{k}^{\prime}}{y_{n-1}^{\prime}}+\cdots,
\end{aligned}
$$

where the coefficients $p_{i k}, q_{i k}$ are given by the equations: 


$$
\begin{gathered}
a_{00} a_{n n} p_{i k}=\sum_{r, s=1}^{n-1} a_{r i} a_{s k} \lambda_{r s} \quad(i, k=1, \cdots, n-1) ; \\
a_{n n} a_{0, n-1} q_{i k}=\sum_{r, s=0}^{n-2} \alpha_{r i} \alpha_{s k \mu_{r s}} \quad(i, k=0,1, \cdots, n-2), \\
\alpha_{00}=-h a_{00}, \quad \alpha_{i 0}=0, \quad \alpha_{0 i}=a_{n-1, i}-h a_{0 i}, \quad \alpha_{r i}=a_{r i}, \\
\mu_{00}=\mu_{n-1, n-1}, \quad \mu_{0 r}=\mu_{r 0}=\mu_{n-1, r}=\mu_{r, n-1}(i, r=1, \cdots, n-2) .
\end{gathered}
$$

Let

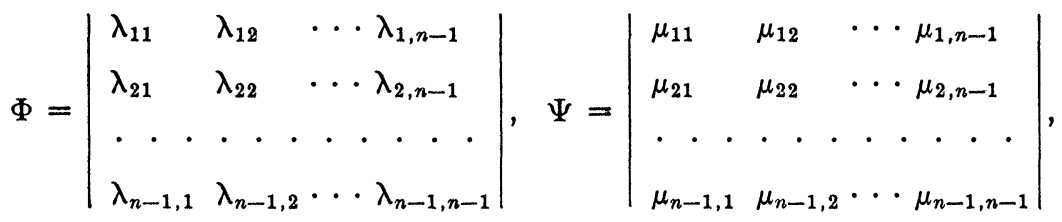

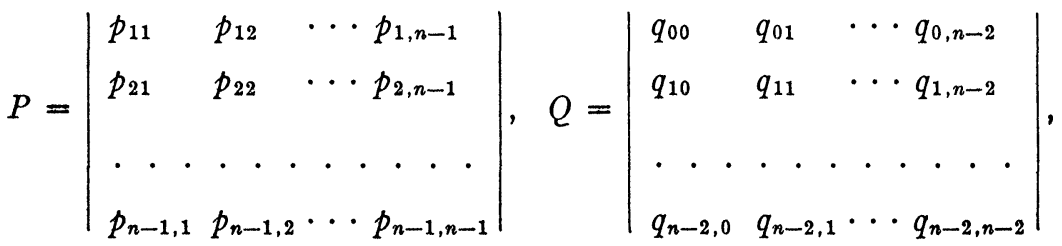

then from equations (17), (18), (21), (22), (23) we obtain

$$
a_{00}^{n-1} a_{n n}^{n-1} P=a_{n-1, n-1}^{2} \Delta^{2} \Phi, \quad a_{n n}^{n-1} a_{0, n-1}^{n-1} Q=h^{2} a_{00}^{2} \Delta^{2} \Psi .
$$

Making use of the result obtained in $\$ 1$ and observing equations (19), (20) we see that the projective invariant $I$ associated with the hypersurfaces $V_{n-1}, V_{n-1}^{*}$ at the points $O, O^{*}$ is

$$
I=\frac{P}{Q}\left(\frac{q_{00}}{p_{n-1, n-1}}\right)^{(n+1) / 8} .
$$

Furthermore, substituting (21), (22), (24) in (25) and reducing by equations (17) it follows that the invariant I now takes the form

$$
I=\frac{\Phi}{\Psi}\left(\frac{\mu_{n-1, n-1}}{\lambda_{n-1, n-1}}\right)^{(n+1) / 8} .
$$

Let $K, K^{*}$ be the curvatures of the hypersurfaces $V_{n-1}, V_{n-1}^{*}$ at the points $O, O^{*}$; and $R, R^{*}$ the curvatures at the points $O, O^{*}$ of the plane sections of the hypersurfaces $V_{n-1}, V_{n-1}^{*}$ made by the plane of the line $O O^{*}$ and the normal to the common tangent hyperplane $t_{n-1}$ at any point on the line $O O^{*}$. By a known formula it is easy to 
demonstrate that

$$
K / K^{*}=\Phi / \Psi, \quad R / R^{*}=\lambda_{n-1, n-1} / \mu_{n-1, n-1},
$$

and therefore that

$$
I=\frac{K}{K^{*}}\left(\frac{R^{*}}{R}\right)^{(n+1) / 8} .
$$

Hence we have the following theorem.

THEOREM. Let $V_{n-1}, V_{n-1}^{*}$ be two hypersurfaces in a space $S_{n}$ of $n$ dimensions having a common tangent hyperplane $t_{n-1}$ at two ordinary point $O, O^{*} ; K, K^{*}$ the curvatures of the hypersurfaces $V_{n-1}, V_{n-1}^{*}$ at the points $O, O^{*}$; and $R, R^{*}$ the curvatures at the points $O, O^{*}$ of the plane sections of the hypersurfaces $V_{n-1}, V_{n-1}^{*}$ made by the plane of the line 0O* and the normal to the common tangent hyperplane $t_{n-1}$ at any point on the line OO*. Then $\left(K / K^{*}\right)\left(R^{*} / R\right)^{(n+1) / 3}$ is a projective invariant associated with the hypersurfaces $V_{n-1}, V_{n-1}^{*}$ at the points $O, O^{*}$.

\section{Chapter II. Two hypersurfaces With DIstinct TANGent} HYPERPLANES AT TWO ORDINARY POINTS

4. Derivation of invariants. Let $V_{n-1}, V_{n-1}^{*}$ be two hypersurfaces in a space $S_{n}$ of $n$ dimensions such that the tangent hyperplanes $t_{n-1}, t_{n-1}^{*}$ at two ordinary points $O, O^{*}$ are distinct, and the common tangent flat space $t_{n-2}$ of $t_{n-1}, t_{n-1}^{*}$ contains the line $O O^{*}$. If we choose the points $O, O^{*}$ to be the vertices $(0,1,0, \cdots, 0),(0, \cdots, 0,1,0)$ of a homogeneous projective coordinate system of reference, and the tangent hyperplanes $t_{n-1}, t_{n-1}^{*}$ to be the coordinate hyperplanes $x_{1}=0$, $x_{n+1}=0$ respectively, then the power series expansions of the hypersurfaces $V_{n-1}, V_{n-1}^{*}$ in the neighborhood of the points $O, O^{*}$ may be written in the form

$$
\begin{aligned}
& V_{n-1}: \frac{x_{1}}{x_{2}}=\sum_{i, k=3}^{n+1} l_{i k} \frac{x_{i}}{x_{2}} \frac{x_{k}}{x_{2}}+\cdots, \\
& V_{n-1}^{*}: \frac{x_{n+1}}{x_{n}}=\sum_{i, k=1}^{n-1} m_{i k} \frac{x_{i}}{x_{n}} \frac{x_{k}}{x_{n}}+\cdots
\end{aligned}
$$

Considering the most general projective transformation of coordinates which shall leave the points $O, O^{*}$ and the hyperplanes $t_{n-1}$, $t_{n-1}^{*}$ unchanged, we may easily show as in $\$ 1$ that the quantities

$$
I=\frac{L M l_{n n} m_{22}}{L_{n+1, n+1} M_{11}}, \quad J=\left(\frac{M}{L}\right)^{n-3}\left(\frac{L_{n+1, n+1} m_{22}}{M_{11} l_{n n}}\right)^{n+1}
$$


are projective invariants determined by the neighborhood of the second order of the hypersurfaces $V_{n-1}, V_{n-1}^{*}$ at the points $O, O^{*}$, where $L_{n+1, n+1}$, $M_{11}$ are respectively the minors of $l_{n+1, n+1}, m_{11}$ in the determinants

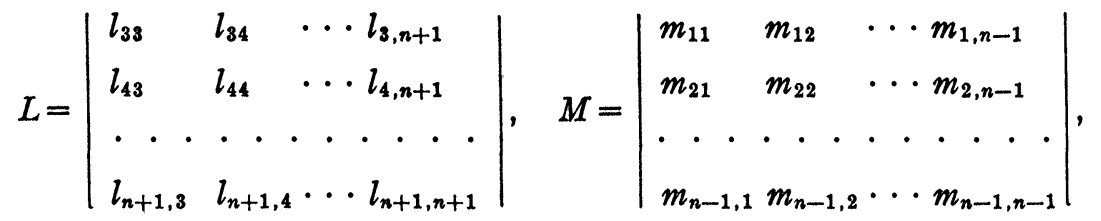

and $L^{\prime}, M^{\prime}, L_{n+1, n+1}^{\prime}, M_{11}^{\prime}$ are denoted by similar expressions.

5. Projective characterizations of the invariants $I, J$. By suitable choice of the system of reference the invariants $I, J$ of equations (31) can be simplified. In fact, if we choose $n-1$ vertices of the system in the common tangent flat space $t_{n-2}$, and the other two $O_{n+1}(0, \cdots, 0,1), O_{1}(1,0, \cdots, 0)$ respectively on the polars $t, t^{*}$ of the flat space $t_{n-2}$ with respect to the asymptotic hypercones of the hypersurfaces $V_{n-1}, V_{n-1}^{*}$ at the points $O, O^{*}$, the invariants $I, J$ then take the simple form

$$
\begin{aligned}
& I=l_{n n} l_{n+1, n+1} m_{11} m_{22}, \\
& J=\left(\frac{L_{n+1, n+1}}{M_{11}}\right)^{4}\left(\frac{m_{11}}{l_{n+1, n+1}}\right)^{n-3}\left(\frac{m_{22}}{l_{n n}}\right)^{n+1} .
\end{aligned}
$$

It should be noticed that the invariant $J$ is reduced to 1 as $n=3$.

The polars $t, t^{*}$ determine a space $S_{3}$, which intersects the hypersurfaces $V_{n-1}, V_{n-1}^{*}$ in two surfaces $S, S^{*}$. These two surfaces $S, S^{*}$ are evidently in the class considered by Buzano and Bompiani, and the corresponding invariant may be easily found from Bompiani's note [1] to coincide just with the invariant $I$. Thus we reach the conclusion:

The invariant I associated with the hypersurfaces $V_{n-1}, V_{n-1}^{*}$ at the points $O, O^{*}$ is the invariant of Buzano at the points $O, O^{*}$ of the surfaces $S, S^{*}$ in which the hypersurfaces $V_{n-1}, V_{n-1}^{*}$ are intersected by the space $S_{3}$ determined by the polars $t, t^{*}$.

To characterize projectively the other invariant $J$ we consider any hyperplane $\pi_{\alpha}$ through the common tangent flat space $t_{n-2}$ :

$$
x_{n+1}=\alpha x_{1} \quad(\alpha \neq 0),
$$

which intersects the hypersurfaces $V_{n-1}, V_{n-1}^{*}$ in two hypersurfaces $V_{n-2}, V_{n-2}^{*}$ of $n-2$ dimensions. Since these two hypersurfaces $V_{n-2}$, $V_{n-2}^{*}$ have a common tangent hyperplane at the points $O, O^{*}$ we may 
determine an invariant, denoted by $I_{\alpha}$, as in $\$ 1$ :

$$
I=\alpha^{2(n-8) / 3} \frac{L_{n+1, n+1}}{M_{11}}\left(\frac{m_{22}}{l_{n n}}\right)^{n / 3} \text {. }
$$

On the other hand, it is useful to consider the hypercones $C, C^{*}$ projecting respectively from the vertices $O_{1}(1,0, \cdots, 0)$, $O_{n+1}(0, \cdots, 0,1)$ the asymptotic hypercones at the points $O, O^{*}$ of the hypersurfaces $V_{n-1}, V_{n-1}^{*}$. These two hypercones $C, C^{*}$ determine a pencil of hyperquadrics in the space $S_{n}$, among which there exist $n-1$ hypercones, two of them being $C, C^{*}$. The line $O_{1} O_{n+1}$ intersects each of the other $n-3$ hypercones in a pair of points. Let $Q_{i}(i=1, \cdots, n-3)$ be any one of each pair of these points, $P$ the point of intersection of the line $O_{1} O_{n+1}$ with the hyperplane $\pi_{\alpha}$, and $D_{i}$ the cross ratio of the four points $O_{1}, O_{n+1}, Q_{i}, P$ on the line $O_{1} O_{n+1}$; then it follows that the invariant $J$ can be expressed in terms of the invariant $I_{\alpha}$ and the $n-3$ cross ratios $D_{1}, D_{2}, \cdots, D_{n \rightarrow s}$ as follows:

$$
J=I_{\alpha}^{3}\left(D_{1} D_{2} \cdots D_{n-8}\right)^{2} .
$$

6. Metric characterizations of the invariants $I, J$. For the purpose of finding simple metric characterizations of the invariants $I, J$, we choose an orthogonal Cartesian coordinate system in such a way that the point $O$ is the origin, the line $O O^{*}$ is the $X_{n-1}$-axis, and the tangent hyperplane $t_{n-1}$ is the coordinate hyperplane $X_{1}=0$. Then the power series expansions of the hypersurfaces $V_{n-1}, V_{n-1}^{*}$ in the neighborhood of the points $O, O^{*}$ may be put into the form

$$
\begin{aligned}
V_{n-1}: X_{1}= & \sum_{i, k=2}^{n} \lambda_{i k} X_{i} X_{k}+\cdots \\
V_{n-1}^{*}: X_{n}= & \mu X_{1}+\sum_{i, k=1}^{n-2} \mu_{i k} X_{i} X_{k}+2 \sum_{i=1}^{n-2} \mu_{i, n-1} X_{i}\left(X_{n-1}-h\right) \\
& +\mu_{n-1, n-1}\left(X_{n-1}-h\right)^{2}+\cdots
\end{aligned}
$$

where $h$ is the distance between the points $O, O^{*}$, and $\mu=\cot \omega, \omega$ being the angle of the tangent hyperplanes $t_{n-1}, t_{n-1}^{*}$.

In order to express the two invariants $I, J$ in terms of the coefficients of expansions (36), (37) we have first as in $\$ 3$ to consider the homogeneous coordinates $y_{0}, y_{1}, \cdots, y_{n}$ of a point defined by formulas (15) and the most general projective transformation of coordinates, which shall leave the point $O$ and the tangent hyperplane $t_{n-1}$ invariant and carry the point $O^{*}$ and the tangent hyperplane $t_{n-1}^{*}$ into the vertex $(0, \cdots, 0,1,0)$ and the coordinate hyperplane 
$y_{n}^{\prime}=0$ of the new coordinate system respectively. An easy calculation, which shall be omitted here, suffices to demonstrate the result as follows:

$$
I=h^{4} \frac{\Phi \Psi \lambda_{n-1, n-1} \mu_{n-1, n-1}}{\Phi_{n n} \Psi_{11}}, \quad J=\left(\frac{\Psi}{\Phi}\right)^{n-8}\left(\frac{\Phi_{n n} \mu_{n-1, n-1}}{\Psi_{11} \lambda_{n-1, n-1}}\right)^{n+1},
$$

where $\Phi_{n n}, \Psi_{11}$ denote respectively the minors of $\lambda_{n n}, \mu_{11}$ in the determinants

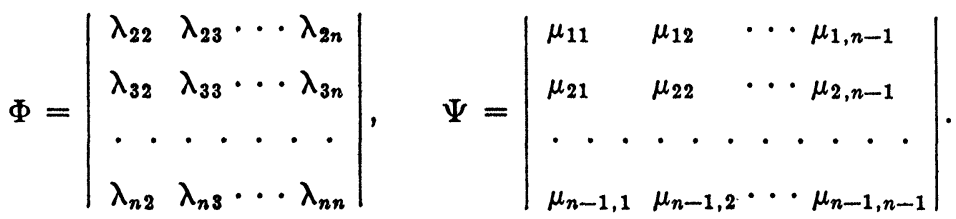

Finally, we shall make use of the normals $O N, O N^{*}$ at the point $O$ of the common tangent flat space $t_{n-2}$ in the tangent hyperplanes $t_{n-1}, t_{n-1}^{*}$. Let $K_{2}, K_{2}^{*}$ be respectively the curvatures at the points $O, O^{*}$ of the plane sections of the hypersurfaces $V_{n-1}, V_{n-1}^{*}$ made by the planes $O O^{*} N^{*}, O O^{*} N$. Further, let $K_{n}, K_{n}^{*}$ be the curvatures of the hypersurfaces $V_{n-1}, V_{n-1}^{*}$ at the points $O, O^{*}$; and $K_{n-1}, K_{n-1}^{*}$ the curvatures at the points $O, O^{*}$ of the hypersurfaces $V_{n-2}, V_{n-2}^{*}$ of $n-2$ dimensions in which the tangent hyperplanes $t_{n-1}^{*}, t_{n-1}$ intersect the hypersurfaces $V_{n-1}, V_{n-1}^{*}$ respectively. Then

$$
\begin{aligned}
K_{n} & =2^{n-1} \Phi, & K_{n}^{*} & =2^{n-1}\left(1+\mu^{2}\right)^{-(n+1) / 2} \Psi, \\
K_{n-1} & =2^{n-2}\left(1+\mu^{2}\right)^{(n-2) / 2} \Phi_{n n}, & K_{n-1}^{*} & =2^{n-2} \Psi_{11}, \\
K_{2} & =2\left(1+\mu^{2}\right)^{1 / 2} \lambda_{n-1, n-1}, & K_{2}^{*} & =2 \mu_{n-1, n-1},
\end{aligned}
$$

and hence we arrive at the following metric characterizations of the invariants $I, J$ :

$$
\begin{gathered}
I=\frac{h^{4}}{16} \frac{K_{n} K_{n}^{*} K_{2} K_{2}^{*}}{K_{n-1} K_{n-1}^{*} \sin ^{2(n-1)} \omega}, \quad J=\left(\frac{K_{n}^{*}}{K_{n}}\right)^{n-3}\left(\frac{K_{n-1} K_{2}^{*}}{K_{n-1}^{*} K_{2}}\right)^{n+1} . \\
\text { BIBLIOGRAPHY }
\end{gathered}
$$

\section{BIBLIOGRAPHY}

1. E. Bompiani, Invarianti proiettivi di una particolare coppia di elementi superficiali del $2^{\circ}$ ordine, Bollettino della Unione Matematica Italiana vol. 14 (1935) pp. 237-243.

2. C. L. Bouton, Some examples of differential invariants, Bull. Amer. Math. Soc. vol. 4 (1898) pp. 313-322.

3. P. Buzano, Invariante proiettivo di una particolare coppia di elementi di superficie, Bollettino della Unione Matematica Italiana vol. 14 (1935) pp. 93-98. 
4. - Interpretazione proiettiva dell'invariante di Mehmke, Bollettino della Unione Matematica Italiana vol. 15 (1936) pp. 173-175.

5. C. C. Hsiung, Projective differential geometry of a pair of plane curves, Duke Math. J. vol. 10 (1943) pp. 539-546.

6. - Projective invariants of a pair of surfaces, Duke Math. J. vol. 10 (1943) pp. 717-720.

7. - On a projective invariant of a certain pair of surfaces, to be published elsewhere.

8. R. Mehmke, Einige Sätze ïber die räumliche Collineation und Affinität, welche sich auf die Krümmung von Curven und Flächen beziehen, Schlömilchs Zeitschrift für Mathematik und Physik vol. 36 (1891) pp. 56-60.

9. - Über zwei die Krümmung von Curven und das Gauss'sche Krümmungsmass von Flächen betreffende charakteristische Eigenschaften der linearen Punkttransformationen, ibid. vol. 36 (1891) pp. 206-213.

10. C. Segre, Su alcuni punti singolari delle curve algebriche, et sulla linea parabolica di una superficie, Rendiconti dei Lincei (5) vol. 6 (1897) pp. 168-175.

11. A. Terracini, Densita di una corrispondenza di tipo dualistico, ed estensione dell'invariante di Mehmke-Segre, Atti Accad. Sci. Torino vol. 71 (1936) pp. 310-328.

National University of Chekiang 